\title{
SYNTHESIS OF SUSTAINABLE DEVELOPMENT SCENARIOS OF SOCIAL ECONOMIC SYSTEMS
}

\author{
Dmitry A. Kononov and Vladimir V. Kul'ba \\ Institute of Control Sciences \\ of Russian Academy of Sciences \\ 65, Profsoyuznaya str. Moscow, Russia, 117806, \\ E-mail: kulba@ipu.rssi.ru
}

\author{
Jana Bikovska \\ Riga Technical University \\ 1 Kalku Street \\ Riga, Latvia, LV-1658 \\ E-mail: jana@itl.rtu.lv
}

\section{KEYWORDS}

Scenario, control, sustainable development.

\begin{abstract}
This paper presents basic principles of a scenario approach application to the problem of optimal development of social economic systems. The formalized description of scenario of system sustainable development is introduced and synergetic and attractive scenarios are examined. The modes providing stable and safe development of social economic system are investigated for synergistic scenarios, however for attractive scenarios it is the control purpose. Scenario design example based on the use of the social economic system development model represented by signed the graph language are given. Formal methods of such scenarios development, the "Object-subject" method and the method of base scenarios, are proposed.
\end{abstract}

\section{INTRODUCTION}

The analysis of the behavior of dynamic processes, appearing in socioeconomic systems under the influence of disturbances of different nature, is an important step of formal decision-making procedures for a wide range of control problems in the systems for providing predetermined security level. Typical tasks of this kind are tasks that appear under investigation of not quite well-structured and non-structured problems with low quality of the initial data and with qualitative description of dependences. Getting a numerical solution with the precise normative models in this case is unreasonable. It is applied in full to the problem of working out of a strategy of regional, national and international crisis-free development. Under such conditions the role of the analysis methods that allow by information available about structural features of an investigated system to judge about appropriate development directions of a dynamic processes, their sustainability and other features, becomes very important. Such a task can be solved by synthesis of scenario of a social economic system (SES) development (Kononov et al. 1999).

Recently, the signed graphs have become a popular tool for analyzing SES functioning and control. They allow working with quantitative and qualitative data and can be effectively applied for a system behavior scenario synthesis. The signed graph model extension to the directed operator graph model allows one to formally develop synergetic and attractive scenarios. They are hypothetic discrete movement trajectories of the modeled system in a phase space of variables (factors), based on the information about system structure and control actions performed (Kononov et al. 2001). The proposed method consists of an approximation of SES development trends by trajectory fragments, defined by impulse processes on the directed graph. Here the basic tendencies of the system development are defined without external influences (synergetic scenarios) or with predetermined control influences (attractive scenarios - direct control task).

If the observed parameter characteristics are negative, it is possible to solve a reverse task - to find effective control decisions and their application to the model (generation of attractive scenarios). As a possible option, static or dynamic control approaches could be applied. The static control approach does not change relations between structural elements; however, the dynamic control approach changes the model structure of SES development.

\section{THE CONCEPT OF A SUSTAINABLE DEVELOPMENT SCENARIO}

The problem of complex systems safety control appears in different fields and it relates to the development of system object of different levels. There are many different kinds of complex systems that could be classified by fields of activity, applied research methods, possibility of application and consequences of one or another control influence, ranging from a particular individual to the objects of global scale. A major feature of SES is absence of an emergency situation during its functioning and development process.

Safety as an aggregation of system functioning properties, including stability, adaptability, vitality, etc., can be formally described by the presence of the following conditions:

- possibility of determination and retention of some system parameters in the defined area of space, i.e. observability and controllability; 
- exclusion of crisis situation appearance in SES that could destroy its elements and/or relations between them;

- possibility of guaranteeing the movement of an object within the phase space in the set direction, for example, in the direction of the strong and balanced growth, i.e. the problem statement and its effective solution about termination, sequencing or continuation of a system behavior scenario along the defined trend.

From the scenario-based research point of view, the safety can be formalized in terms of events and situation.

Expected event of a system behavior is defined as $\mathfrak{I}=(\mathbf{x}(t), \mathbf{y}(t), t)$, where $t$ - a time instant, $\mathbf{z}(t)=(\mathbf{x}(t), \quad \mathbf{y}(t))-$ expected realization of the environmental parameters and the phase trajectory.

Situation $S(t)$ at the time instant $t$ is defined as a set of events which occurred before the moment $t$ :

$$
\begin{aligned}
& S(t)=\left\{\mathfrak{J}^{(i)}\left(\mathbf{x}^{(i)}\left(t_{i}\right), \mathbf{y}^{(i)}\left(t_{i}\right), t_{i}\right)\right. \\
& \left.0 \leq t_{i} \leq t, \quad i=0,1, \ldots, s ; t_{0}=0\right\}
\end{aligned}
$$

Condition $I(t)$ at time instant $t$ is defined as $(S(t), \Theta(t))$, where $S(t)$ - situation at time instant $t, \Theta(t)$ - quasi-informational hypothesis of a decision-making person.

Scenario $\mathfrak{R}$ of system behavior is defined as a pair $\left(I\left(t_{i}\right), t_{i}\right)$, composed according to $A^{(t)}$ choice law:

$$
\mathfrak{R}=\mathfrak{R}\left\{\left(I\left(t_{i}\right), t_{i}\right)\right\} \quad i=0,1, \ldots, N ; t_{0}=0 .
$$

The value $N$ is called scenario depth, and $T=t_{N}$ scenario horizon.

To apply the scenario-based research SES it is important to select the events significant to an expert which determine the features of a model of fixing of events in the set subject domain on the base of the special knowledge of the experts (Kononov and Kul'ba 1996).

Definition 1. Situation $S(t)$ is called crisis-free, if

$$
\mathbf{z}(t) \in \operatorname{int} \hat{Q}
$$

for all events from $S(t)$ significant to expert, i.e. the generated sequence of the states is within the set of $\hat{Q}$ possible states.
The definition of a crisis-free situation dependent on the way of determining $\widehat{Q}$ could be different.

Let a scenario $\mathfrak{R}$ with horizon $T$ be defined.

Definition 2. Scenario $\mathfrak{R}$ is called the scenario of sustainable development, if for any $\tau=\overline{0, T}$ situation $S(\tau)$ is crisis-free.

The scenarios of sustainable development are characterized by the most desirable methods of SES development, saving its homeostasis.

\section{SCENARIO-BASED RESEARCH OF SUSTAINABLE DEVELOPMENT IN THE MODELS OF SES BEHAVIOR WITH SIGNED DIGRAPH}

Let us consider a scenario research of SES when its behavior model is introduced as an operator graph. A dynamic of the system behavior is set on the graph $G(X, E)$ where in each node $x_{i} \in X \quad(i \in \widehat{N}=\overline{1, n})$ the parameters' column $\quad \mathbf{v}^{(i)} \in E^{P} \quad(i \in \widehat{N})$, which determines a current state of the system, is defined.

At time instant $t=0$ parameters have initial value $\mathbf{v}^{(i)}(0)$. Graph $G(X, E)$ and a set $\widehat{F}=\left\{\bar{F}^{(p)} \quad 1 \leq p \leq P\right\}$ of adjacency matrix $\bar{F}^{(p)}$ define structure of the transient, which can be observed as a sequence of transformations of a condition of system parameters by the rule:

$$
\mathbf{v}_{p}(t)=\zeta^{(p)}\left(\widehat{F}, \vartheta, \mathbf{v}_{p}(t-1)\right) \text { where } 1 \leq p \leq P,
$$

where $\mathbf{v}_{p}(t)=\left\{v_{p}^{(1)}(t), v_{p}^{(2)}(t), \ldots, v_{p}^{(n)}(t)\right\}$ is a vector - line of values of the parameter $p$, observed in nodes of the directed graph at time instant $t ; \vartheta$ detected or applied control influence on SES elements.

An operator digraph, in which intermatrix relations $\bar{F}^{(p)}$ in the operator $\zeta^{(p)}$ are missing, is called operator digraph with independent components (ICoperational graph). IC-operator graph describes structure and interaction of base elements of SES, as a rule, over the range of one stratum fields of the description and control.

The sequence of change of parameter $\vartheta$ over time leads to a sequence of changes of a condition of the system parameters $\mathbf{V}^{(i)}$ that form the determined step-to-step scenario $\mathfrak{R}$. 
The multitude of scenarios of SES behavior on operator graph is determined by the strategy of its formation. Two types of such strategy are considered: operationally-tactical - $k$-step sequence of impulses $\operatorname{Im}(t, k)=\left\{O I_{0}^{(i)}(\tau)_{i \in \hat{N}}\right\}_{(\tau=\overline{t, t+k})}$ and strategic - $k$ step sequence of SES structural transformations $\Phi(t, k)=\{A(\tau)\}_{(\tau=\overline{t, t+k})}$, that starts at time instant $t$.

To each of the chosen sequences there corresponds the linear time step-to-step scenario as a time sequence of step-to-step events significant to expert.

For the IC-digraph $G(X, A, \zeta)$ on the horizon $T$ the sequence $\Phi(0, T)$ is constant, i.e. $A(\tau)=A$ for all $\tau=\overline{0, T}=\widehat{T}$, and $T$-step impulse process is defined as follows:

$$
\operatorname{Im}(0, T)=\left\{\mathbf{O I}_{0}(\tau)=\left(O I_{0}^{(i)}(\tau)\right)_{i \in \hat{N}}\right\}_{(\tau \in \hat{T})} .
$$

It is by virtue of a dynamic model

$$
\begin{aligned}
& \mathbf{O I}(\tau)=\mathbf{O I}(\tau-1) A+\mathbf{O I} \mathbf{I}_{0}(\tau)(\tau=0,1, \ldots), \\
& \Delta \mathbf{v}(\tau)=\mathbf{v}(\tau)-\mathbf{v}(\tau-1)=\mathbf{O I}(\tau-1) A+\mathbf{O I}_{0}(\tau),
\end{aligned}
$$

where $\mathbf{O I}(\tau)$ is the impulse, which has been cumulated up to the time instant $\tau$, corresponds to the determined time step-to-step scenario with the beginning in the expert significant event $\mathbf{v}(0)$ :

$$
\mathfrak{R}(0, T, \mathbf{v}(0))=V(\hat{T})=\left\{\mathbf{v}(\tau)=\left(v^{(i)}(\tau)\right)_{i \in \hat{N}}\right\}_{(\tau \in \hat{T})}
$$

By a threat the presence of a hypothetical opportunity of realization of the undesirable phenomena could be called. Describing SES as a formal system object we will define a threat as a set of system and environment factors that could lead to deterioration of a condition of its system parameters from the decision-maker point of view. There are internal and external threats. For impulse processes on operator graph the following definitions are given:

The set of available impulses is given $\Omega \mathbf{I m} \in E^{n}$.

Definition 3. $T$-step impulse process $\operatorname{Im}(0, T)$ is called admissible, if

$$
\mathbf{O I}_{0}(\tau) \in \Omega \mathbf{I m} \text { for all } \tau \in \hat{T} .
$$

Definition 4. At realization of admissible impulse process $\operatorname{Im}(0, T)$ conditions $\mathbf{v}(0)$ contain $T$-step threat, if

$$
\mathbf{v}(T+1) \notin \operatorname{int} \widehat{Q}(\tau+1) .
$$

Definition 5. Condition $\mathbf{v}(0)$ is called $T$ - critical if it contains $T$-step threat at realization of any admissible impulse process

$$
\mathbf{v}(T+1) \notin \operatorname{int} \hat{Q}(\tau+1) .
$$

A 1-critical conditions is called emergency.

The scenario-based research of safe development of a system (3)-(6) can be carried out on the base of various models depending on the sets of allowable conditions $\hat{Q}(\tau)$ and strategy of behavior, which are permissible impulse processes.

\section{THE MODEL OF KYOTO PROTOCOL}

As an example of the signed digraph methodology application, the mechanism of ecological risk management based on the market distribution of quotas of environment pollution among countries-pollutants is considered.

According to the international contract, there is a mechanism of strict regulations of cumulative emission of harmful substances in an environment from the side of all industrial enterprises of the countries-participants of the contract (the Kyoto Protocol). It means that each participant can get in the world market a quota on a manufacturing pollution in a certain amount. In abstract case of the model, it is considered that if the participant for some reasons exceeds the quota limits provided his work is stopped. As the quotas distribution is a market mechanism, quotas can also be objects of sale and purchase. A competitive struggle can be developed around quotas. It is essential that the level that limits cumulative pollution issue is not defined by a demand for quotas in the market - international agreements determine them.

As the base processes that describe the considered mechanism, two subsystems are considered for their qualitative analysis:

1) subsystem "Socium":

- change of an environment quality;

- change of a life quality;

- change of risk of an occurrence of an extreme ecological situation.

2) subsystem "Industry":

- industry growth;

- change of the level of competitive struggle for pollution quotas.

The following parameters describing phase states of base processes are considered:

$\mathrm{Q}$ - population life quality;

PO - number of inhabitants;

$\mathrm{W}$ - number of workplaces; 
I - pollution level;

$\mathrm{H}$ - risk of occurrence of an extreme ecological situation;

$\mathrm{N}$ - enterprise number;

$\mathrm{C}$ - level of competitive struggle;

$\mathrm{P}$ - the price on generalized resources - quotas;

E - efficiency of use of the generalized resource;

M - allowed marginal level of issue of harmful substances.

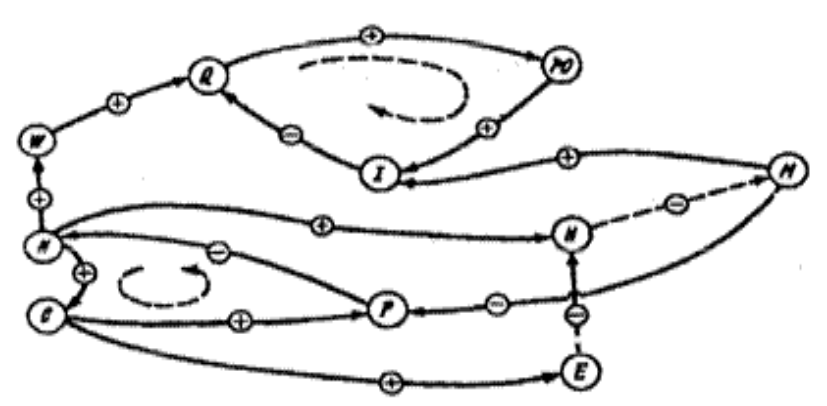

Figure 1: Graph of "Kyoto" Model

The system model is constructed as a signed digraph (see Figure 1), where nodes are the base processes and parameters that characterize them. The interference of processes determined by the socioeconomic and natural laws is represented by realization of edges with the appropriate signs. At construction of the model it is necessary to take into account time parities (ratio) between the basic and caused impulse processes. In the model, under consideration an influence transfer time between base processes is accepted to be equal to the length of the appropriate way between them in the signed digraph.

For the constructed model, sufficient conditions of impulse stability are unsatisfied. It means that at occurrence of an impulse in any node of $(\mathrm{N}, \mathrm{C}, \mathrm{P})$ cycle there is a linear increase in the module of an impulse in nodes of a cycle $(\mathrm{Q}, \mathrm{PO}, \mathrm{I})$, i.e. a linear resonance. This phenomenon can have the following interpretation: fluctuations in amount of the enterprises conduct the fluctuations in an occupation level of the population that "shakes" processes of migration. Increasing fluctuations on amplitude in a population attract growth of environmental contamination due to household pollution. Computing experiment shows the increase in fluctuations amplitude.

Assume that socium cannot remain indifferent to changes of risk of extreme ecological situation. Within the framework of the offered formalized model it is possible to use various alternatives of operationallytactical and strategic management. As strategic management it could mean drawing an edge or further edges from the node $\mathrm{H}$. The solution when the negative edge $(\mathrm{H}, \mathrm{M})$ is added to the model could be considered as reasonable in case of risk increase of emergency solution.
In this case it also does not solve the problem: a resonance takes place again. Practically it means that in any possible way it is reasonable to complicate influence of rival elements on market mechanisms of ecological risk management.

Investigation of direct impulse and structural control approaches enables one to get typical modes of system behavior with simulation model.

\section{CONSTRUCTION OF THE SCENARIOS OF SUSTAINABLE DEVELOPMENT WITH THE "OBJECT-SUBJECT" METHOD}

This section discusses the method of optimal control of the distribution process of indignations by allocation of nodes-objects and nodes-subjects. The node-object corresponds to a parameter, whose value has to be controlled. The node-subject corresponds to a parameter, by influencing which this controlled change outstripping in time is carried out. Control is accomplished by means of preventive inclusion into nodes-subjects a sequence of external impulses that form the attractive adaptive scenario of safe development $\mathfrak{R}^{(\mathrm{sec})}\left(T^{(\mathrm{sec})}\right)$ on the horizon $t \in\left[t_{0}, t_{1}\right]=T^{(\mathrm{sec})}$. At construction of the safe development scenario $\mathfrak{R}^{(\mathrm{sec})}\left(T^{(\mathrm{sec})}\right)$ it is necessary to specify:

- adaptive strategy construction factors:

- a set $\mathbf{g}=\left\{g_{j} \in \hat{N}, j=\overline{1, g}\right\}$ of nodes-objects, so $V_{o b}=\left\{v^{(l)} \quad l \in \mathbf{g}\right\} \in R^{g}$ - set of values of their parameters;

- allowable areas $Q_{o b}\left(t^{(l)}\right)(l \in \mathbf{g})$ of values of the given characteristic $\mathbf{h}\left(V_{o b}, t\right) \in Q_{o b}\left(t^{(l)}\right)$ nodesobjects $V_{o b}$ at time instant $t$;

- a set of time moments $T^{(l)} \subseteq T^{(\mathrm{sec})} \quad(l \in \mathbf{g})$ in which restrictions for values of parameters in tops - objects are entered;

- control subjects:

- set $\mathbf{q}=\left\{q_{j} \in \widehat{N}, j=\overline{1, q}\right\}$ of nodes-subjects, so $V_{u}=\left\{v^{(u)} \quad u \in \mathbf{q}\right\} \in R^{q}$ - set of values of their parameters;

- set of time moments $T^{(u)} \subseteq T^{(\mathrm{sec})} \quad(u \in \mathbf{q})$ influences on nodes-subjects;

- sequence of operational and tactical impulses in node $u \in \mathbf{q}$

$$
\mathbf{I m}^{(u)}\left(T^{(u)}\right)=\left\{O I^{(u, 0)}(\tau)\right\}_{\left(\tau \in T^{(u)}\right)} \in Q^{(u)}\left(\mathbf{I m}\left(T^{(u)}\right)\right),
$$

concentrated in the set of allowable impulses $Q^{(u)}\left(\operatorname{Im}\left(T^{(u)}\right)\right)$; 
- control purpose:

deduction of values of the parameters $V_{o b}$ appropriate to nodes-objects, in the allowed area

$$
h^{(l)}\left(V_{o b}, t\right) \in Q_{o b}\left(t^{(l)}\right) \text { at } t^{(l)} \in T^{(l)}
$$

for all $l \in \mathbf{g}$.

Thus, the control task is formulated as follows: the initial expert significant event $\mathbf{v}\left(t_{0}\right)$ is defined. It is necessary to construct the attractive $T$-step-to-step scenario $\mathfrak{R}^{(\mathrm{sec})}\left(T^{(\mathrm{sec})}\right)$ of safe development, i.e. to carry out synthesis of optimum control (10) as a sequence of $T$-step-by-step expert significant events $\mathfrak{R}^{(\mathrm{sec})}\left(T^{(\mathrm{sec})}\right)=\mathfrak{R}^{(\mathrm{sec})}\left\{\left(I^{(\mathrm{sec})}(t), t\right)\right\} \quad t \in T^{(\mathrm{sec})}$,

satisfying conditions (11). This task is a reverse control task.

Let's consider solving the task under the following conditions:

$$
\begin{aligned}
& \mathbf{h}\left(V_{o b}, t\right)=\mathbf{v}(t)-\mathbf{v}(t-1) \quad t \in T^{(\mathrm{sec})}, \\
& Q_{o b}\left(t^{(l)}\right)=\left\{v^{(l)}(t) \mid h^{(l)}\left(V_{o b}, t\right) \leq 0\right.
\end{aligned}
$$

at $t^{(l)} \in T^{(l)}$ for all $\left.l \in \mathbf{g}\right\}$,

that describes preservation (not increase) of the parameter values in the given area for nodes-objects at certain time moments $T^{(l)}$;

$Q^{(u)}\left(\mathbf{I m}\left(t_{0}, t_{1}\right)\right)=\mathbf{L}_{\mathbf{q}}\left(\mathbf{I m}\left(t_{0}^{(u)}, t_{1}^{(u)}\right)\right)$

$u \in \mathbf{q}, t^{(u)} \in T^{(u)}$,

i.e. control tools are any impulse processes begun in nodes-subjects $\mathbf{q}$ on the interval $T^{(u)}$.

It is possible to show that for processes (3)-(6) the following holds:

$\mathbf{v}(t)-\mathbf{v}(t-1)=\sum_{\tau=0}^{t} \mathbf{O I}_{0}(\tau) A^{t-\tau}$ for

$t=1,2 \ldots$, or

$v^{(l)}\left(t^{(l)}\right)-v^{(l)}\left(t^{(l)}-1\right)=\sum_{u=1}^{n} \sum_{\tau=0}^{t^{(l)}} O I_{0}^{(u)}(\tau) a_{u l}^{t^{(l)}-\tau}$, where $a_{j l}^{t-\tau}$ is the $(j, l)$-th element of the matrix $A^{t-\tau}$. Hence, conditions (13)-(14) will be transformed to

$$
\sum_{\tau=0}^{t^{(l)}} \sum_{u=1}^{n} O I_{0}^{(u)}(\tau) a_{u l}^{t^{(l)}-\tau} \leq 0 \text { for all } l \in \mathbf{g}
$$

or

$$
-\left(\sum_{u \in \mathbf{q}} \sum_{\substack{0 \leq \tau \leq t \\ \tau \notin T^{(u)}}} O I_{0}^{(u)}(\tau) a_{u l}^{t^{(l)}-\tau}+\right.
$$

$\left.\sum_{u \notin \mathbf{q}} \sum_{\tau=0}^{t^{(l)}} O I_{0}^{(u)}(\tau) a_{u l}^{t^{(l)}-\tau}\right)=b^{(l)} \quad l \in \mathbf{g}$

Thus, the solving of the stated reverse task is reduced to solving of system of linear inequalities with regard to variables $O I_{0}^{(u)}(\tau)\left(u \in \mathbf{q}, t \in T^{(u)}\right)$ :

$$
\sum_{u \in \mathbf{q}} \sum_{\substack{0 \leq \tau \leq t \\ \tau \in T^{(u)}}} O I_{0}^{(u)}(\tau) a_{u l}^{t^{(l)}-\tau} \leq b^{(l)} \quad l \in \mathbf{g}
$$

\section{SYNTHESIS OF SCENARIOS \\ OF SUSTAINABLE DEVELOPMENT BY THE METOD OF BASE SCENARIOS}

The method of optimum control of indignation distribution process by distinguishing nodes-objects and nodes-subjects assumes that the area of operating influences is a linear space, i.e. is calculated on use of infinite admissible pulse processes, which in real life is hardly possible. Besides, the method of its decision has an essential shortcoming: the received SES sequence, though the scenario of $T$-safe development, but for any $\delta>0$ contains $\delta$-step threat. At the same time on the base of the results described in (Kononov et al. 2007) the following problem can be solved.

The initial expert significant event $\mathbf{v}_{0}(t)$ is set. To construct an attractive scenario of $\delta$-sustainable $T$-safe development $\mathfrak{R}^{(\mathrm{sec})}\left(T^{(\mathrm{sec})}, \delta\right)$, i.e. to carry out synthesis of the optimum scenario in the form of sequence step-to-step events significant to experts $\mathfrak{R}^{(\mathrm{sec})}\left(T^{(\mathrm{sec})}, \delta\right)=\mathfrak{R}^{(\mathrm{sec})}\left\{\left(I^{(\mathrm{sec})}(t), t\right)\right\} \quad t \in T^{(\mathrm{sec})}$, satisfying condition (9).

The mathematical reason to use the method consists in the following theorems.

Theorem 1 (on the splitting of the expanded phase space). On directed graph of the dimension $n$ for each matrix $A$ there exists a finite canonical structure $\Xi(A)$, representing expert-significant partition of the expanded phase space $Z$

$\Xi(A)=\left\{Z^{(k)} \quad k=\overline{1, s}\right\}, Z^{(k)} \cap Z^{(l)}=\varnothing$

with $k \neq l, Z=\sum_{k=1}^{s} Z^{(k)}$.

Each of these elementary partitions $Z^{(k)}$ is absorbent for any independent pulse process that was started in it (Kul'ba et al. 2004).

Thus, for each matrix $A$ it is possible to specify in advance the modes of the SES concentration generated by the pulse processes, i.e. to compute (rather than to 
indicate by expert) expert- significant partition $Z^{(k)}$ $(k=\overline{1, s})$ the expanded phase space $Z$.

The parameters grouping $k$-root subspace, are connected. The specified groups set SES stratification.

Theorem 2 (About basic modes of pulse scenarios of SES development). There are the following types of basic scenarios: resonant development scenario (RDscenario), compressive development scenario (CDscenario), fluctuation development scenario (FDscenario), resonance-fluctuation development scenario (PFD-scenario of type "a untwisted spiral"), compressing-fluctuation development (CFD-scenario of type "a twisted spiral"). It is possible to extract also their extreme cases: the stationary scenario and the reflection scenario.

Theorem 3 (About modes of impulse scenarios of SES development). The expanded phase space can be presented as follows:

$Z=\bigcup_{k=1}^{s} Z^{(k)}, Z^{(k)} \cap Z^{(l)}=\varnothing$ where $k \neq l$,

i.e. $S$ non-crossed subspaces, in each of which basic modes of development SES are defined.

Any step-to-step expert significant event can be represented in the form of

$\mathbf{v}(t)=\sum_{j=1}^{s} \mathbf{v}^{(j)}(t),<\mathbf{v}^{(j)}(t), \mathbf{v}^{(k)}(t)>=0$,

$j \neq k, j, k=\overline{1, s}$,

where $\mathbf{v}^{(j)}(t)$ is concentrated in $j$-th EIE $Z^{(j)}$.

Theorem 4. Any step-to-step determined scenario corresponding to the set of impulse process on constant signed digraph, can be represented as a linear combination of base scenarios of SES development.

The presented theorems allow one to completely solve problems of the reverse scenario control on signed digraphs and to build for their decision effective computing schemes, in particular, for restrictions (11) with various functions $h^{(l)}\left(V_{o b}, t\right)$, and also at the defined sets of allowable impulses $\Omega \mathbf{I m}$.

\section{CONCLUSIONS}

Nowadays sustainable development of social economic system is a primary task of its existence. In the paper is described scenario-based approach to a research of social economic systems behaviour that allows synthesizing synergistic and attractive scenarios of a system development in order to prevent and control possible emergency situations. As the example, a model of the Kyoto Protocol is developed with signed directed graph and possible control actions and their consequences are discovered.

Contribution of co-author J.Bikovska is supported by the European Social Fund within the National Programme "Support for carrying out doctoral study programs and post-doctoral research" project "Support for the development of doctoral studies at Riga Technical University".

\section{REFERENCES}

Kononov D.A., Kosyachenko S.A., and Kul'ba V.V. 1999. "Analysis of scenarios of development of socioeconomic systems in emergency control systems: models and methods". Avtom. And Rem. Cont., Vol.90. Part 2. No. 9, pp. 1303-1320.

Kononov D.A., Kosyachenko S.A., and Kul'ba V.V. 2007. Design and analysis of development scenarios of social economic systems with the application of the operator graph apparatus, Automation and Remote Control, 2007, vol. 68, No. 1.pp. 109-123.

Kononov D.A. and Kul'ba V.V. 1996. Ecological management: Object development scenarios and control of ecological environment. Inzh. Ekol., No 6.

Kononov D.A., Kul'ba V.V., and Shubin A.N. 2001. Stability of socioeconomic systems: scenario investigation methodology, Preprints Vol., pp.91-96. $8^{\text {th }}$ IFAC Conference of Social Stability: The Challenge of Technological Development; SWISS'01 27-29 Sept. 2001; Vienna, Austria.

Kul'ba V.V., Kononov D.A., Kosyachenko S.A., and Shubin A.N. 2004. Methods of formation of scenarios of development of social and economic systems [in Russian]. AV.A. Trapeznikov Institute of Control Sciences, Russian Academy of Sciences, Moscow.

\section{AUTHOR BIOGRAPHIES}

VLADIMIR V. KUL'BA, Academician, Dr.habil.sc., Professor, Trapeznikov Institute of Control Sciences Russian Academy of Sciences. He has made significant contribution in areas of control theory, optimal modular data processing, local and distributed data bases, network computing systems, and others.

DMITRY A. KONONOV, Dr.Math., Professor, Trapeznikov Institute of Control Sciences, Russian Academy of Sciences, Moscow, Russia. His research area is development of mathematical models and methods for analysis and synthesis of complex systems' scenario.

JANA BIKOVSKA, Mg.sc.ing, Ph.D. student, teaching assistant at the Department of Modelling and Simulation, Riga Technical University, Latvia. Her professional interests are in the field of supply chain management, simulation based business games, scenario approach in complex systems with application to simulation games. 